\title{
Dark modes, slow modes, and coupling in multimode systems
}

\author{
H. Benisty \\ Laboratoire Charles Fabry de l'Institut d'Optique, CNRS, Université Paris-Sud, Campus Polytechnique, RD 128, \\ 91127 Palaiseau Cedex, France (hb@pmc.polytechnique.fr)
}

Received October 27, 2008; accepted December 7, 2008; posted January 27, 2009 (Doc. ID 103323); published March 17, 2009

\begin{abstract}
We present general aspects of coupling among two or more families of modes with a view to deepen the insight on so-called "dark modes." We first review the relationship of dark modes, "coherent population trapping," "rotating wave approximation," "coupled-mode theory," and a few related concepts. The approach we emphasize is related either to inhomogeneous light-matter strong coupling or to the variety of multimode coupled systems designed for slowing down light or for filtering light. Some semantic caveats are discussed, notably down to what can be termed "dark" and "bright" in as simple a system as a distributed Bragg reflector case. A generic " $N-F$ " classification simply states that whatever the total number $N$ of modes, the key point is the number $N_{F}$ of "prediagonal" families, since the number $N_{b}$ of bright modes is simply $N_{F}$ leaving $N_{d}=N-N_{F}$ dark modes. (C) 2009 Optical Society of America

OCIS codes: $130.0130,270.0270,130.5296,140.3945,260.2030$.
\end{abstract}

\section{INTRODUCTION}

A number of recent proposals exploit the concepts of dark modes, either for photonic crystal and photonics use, or, closer to its origin, in the light-matter interaction. The aim of this communication is to deepen the understanding and classify the phenomenology of dark modes arising in $N$-moded ( $N$ levels) systems with $N>2$.

From an optoelectronic perspective, no firm connection to the dark states concept has been formulated. Hence, we start by providing the reader with a review of the concepts history using this terminology. The common mathematical tool of non-Hermitian $N$-level matrices and their SU(3) invariance properties [1-4] is one of the generic similarities allowing the dark states to practically pervade many of the recent fields of photonics.

For our purpose let us focus on the birth in 1976 of the concept of dark states from the experiments by Alzetta et al. [5,6] and Arimondo and Orriols [7], and to the large body of coherent population trapping (CPT) works [8] for which the 1996 review by Arimondo [9] is a welcome entry point. CPT has become a nickname even more popular than dark states for this domain. This semantic shift makes the re-emergence of the same dark states in other topics complex to trace.

As for CPT itself, briefly said, when sending two crossed lasers on a sodium vapor with spatially variable magnetic field, conditions of extinction of the sodium fluorescence were seen macroscopically. The three states in a typical CPT experiment form a so-called " $\Lambda$ " system. The main signature is the disappearance of the absorption (and thus the luminescence) from a laser pumping one "leg" of the $\Lambda$, when a second laser is switched on with the proper strength and tuned around the other leg transition. The most straightforward explanations exploit the "dressed state" picture, whereby under the influence of the intense laser fields, hybrid atom-light states should be employed, as they are known in Rabi oscillations theory of a two-level system. These states split apart from the bare states, so that when one pumps apparently a single leg, what is actually prepared is an atom in a coherent superposition of two atom-field states. If, due to the role of all recombinations not hitherto discussed, all the atomic population is eventually transferred and sheltered in an antisymmetric dressed-atom-light configuration for which spontaneous or stimulated decays are forbidden, a macroscopic "dark" appearance results. The characteristic "peak in the dip" feature of CPT modified absorption can be found by a standard search from the above guidelines in dozens of related recent papers, but it is beyond our scope to bridge more precisely our approach with these flourishing recent developments.

The general framework and usefulness of the rotating wave approximation to describe multilevel atomic systems coupled by laser fields has been addressed, among others by $[3,10]$. The description of the slow time evolution of the diagonal part of the density matrix is the main result that carries a similarity with coupled-mode theory in the domain of waveguides etc. [11-13].

Arimondo [9] further explains the application of the $\mathrm{CPT}$ to amplification without inversion, adiabatic transfer, and velocity-selective CPT for atom cooling. The work of Harris and Imamoglu [14] and Harris [15] has further popularized a particular "variant," the regime of electromagnetically induced transparency or EIT. In generic CPT, there is a need to have two large laser amplitudes, translating into two nonzero Rabi frequencies, so that the dressed states have a particular coupling canceling absorption of one leg. In EIT, bare atom states are dressed by a single laser in such a way that absorption is canceled even for a weak "probe" field of the second laser, providing a kind of linear response, and justifying the name EIT for this variant [16]. This does not preclude the existence of 
an advantageous nonlinear regime based on these EIT conditions [17]. Both nonlinearities and EIT are related to successful ways of strikingly slowing down light, the common point being the strong effect of EIT on the refractive index dispersion $[9,18]$. We leave these issues aside because the concept of dark states has not been so much favored along this wide branch of activity. On the contrary, the domain of exciton physics has been well penetrated by this terminology. References [19,20] represent two examples among other of late 1990s work. Dicke states [21], which have been key to theorizing superradiance [22], are welcome in exciton physics all the more because excitons have readily been a natural vehicle to enhance superradiance or radiative phenomena in general. In this communication, a paper on excitons in microcavities [23] (socalled cavity polaritons [24,25]), subjected to inhomogeneous broadening, will serve as our initial basis to address the generality of dark states.

Also connected to similar ideas is the use, in molecular physics, of dark states to address coupling from a potential surface of an excited state to the vibronic manifold of a lower lying potential surface, nearly a thermodynamic "bath." Hamiltonians similar to those interesting us [23] are, thus, found in [26,27] in the late 1980s. The same partly holds for [28] on Rydberg atoms with locally structured energy landscapes. More recently, related contributions in highly excited collisional effects were also reported [29-31].

Finally, for the sake of completeness, dark states may be not directly relevant to our present work or even misleading: the dark state terminology often denotes a luminescent material irreversible degradation, a case that we discard here. Another use arises from the fundamental description of light-matter interaction, whereby "dark eigenstates" are those of the Hamiltonian before an electromagnetic field term in some gauge is included [32], e.g., $p^{2}$ instead of $(p-q A)^{2}$. Nor does it directly concern us here. We close this "semantic" review with Table 1 which summarizes the above uses.

Let us now turn to the reasons for our contribution, which is the increased use of the dark state or dark mode terminology in the area of waveguides, nanophotonics, photonic crystals, and plasmonics [33-40]. For man-made physical objects such as waveguides instead of atoms, we have to consider modes localized in a specific dielectric (or metallodieletric) structure. Without perturbation (e.g., without periodicity or cavity-waveguide interaction), the sets of eigenmodes would merely suffer from dissipative incoherent losses involving non-Hermitian behavior (quasi-normal modes), but no coupling. To endow some desired optical device function, modes are generically forced to interact through local or periodic perturbations, an interaction that is visually rendered by the typical associated anticrossing patterns in the dispersion. In this context, dark modes denote modes whose interaction is weaker than "expected." This expectation may be seen to some extent as an "educated guess" when many modes are involved.

Consider a system that has $N=N_{p}+N_{q}$ modes (states) that belong to two families $\left(N_{F}=2\right)$. In generic cases anticrossing patterns are described as a function of detuning as an order parameter; in periodic cases a wave vector $k$ additionally labels dispersion branches; for a given $k$, the $N$ branches correspond to $N$ states. Here, there are $N_{p}$ modes in family 1 , and $N_{q}$ in family 2 . All $N_{p}$ modes are coupled to potentially all $N_{q}$ modes, but the main assumption is that the $N_{p}$ modes and $N_{q}$ modes do not couple among themselves, they are "eigenmodes among themselves" (also called "prediagonalized" Hamiltonian, we hypothesize a relation with the invariance subsets of [2]). They can also be thought of as two degenerate subsets of modes, with orthogonality rules inside each subset, plus a variable degree of degeneracy lifting, stemming from inhomogeneous broadening, for example. We will show in Section 2 that this situation will produce $N_{b}$ "bright modes" and $N_{d}=N-N_{b}$ dark modes, namely, $N_{b}=2$ and $N_{d}=N-2$. We then discuss heuristic explanations for this simple result: we provide more insight of the system response in Section 3, based on an analysis of a secular equation in a simple known case [23,27]. We then examine in Section 4 the dispersion relations with two families, and start by the most basic cases $\left(N_{p}=1\right)$, before going to a more general case $\left(N_{p}>1\right)$. Section 5 generalize the results to more than two families of modes, namely, $N_{F}$ families, and proposes that $N_{b}=N_{F}$ and $N_{d}=N-N_{F}$ are the number of bright and dark states in this case. The natural classification emerging from this view could help in labeling the several kinds of dark modes systems encountered in the growing literature on the topic. We finally examine the relationship with slow light of passive linear nature (Section 6), when multimode interactions are met, before concluding (Section 7).

\section{EXAMPLES, ANALOGIES AND CAVEATS FOR DARK MODES}

For example, in our system that has $N=N_{p}+N_{q}$ modesstates, indices $p$ (modes in family 1 ) and $q$ (modes in family 2) may refer to forward and backward modes [41], or to backward and quasi-localized modes such as coupled resonator optical waveguide (CROW) modes [34-37]. Families $p$ and $q$ may also be photon modes and atom-excitonpolaron states (among many others, see [19]), often with negligible dispersion, interacting together. We anticipate that such situations may arise as well in the context of metamaterials since among them the most striking ones, the so-called negative index materials, have internal degrees of freedom that are crucial for their electromagnetic behavior.

As for the bright-dark modes, they refer to the fact that most of a given $\left(p_{j}, q_{k}\right)$ interaction, i.e., a coupling that would produce the second state if excited by the first in isolation, will disappear when the whole bunch of coherent interactions is present-in atom physics, nonlinear effects correspond to this case: light $\rightarrow$ coherent matter polarization $\rightarrow$ coherent light, notably the so-called $\Lambda$ three-level situation. Hence in atom physics a dark state means no absorption-no luminescence instead of an expected bright transition for the isolated $\left(p_{j}, q_{k}\right)$ case. In the middle of an absorption peak, a dip with allowed transmission may occur because one then excites a selfsustained oscillation frequency of the "loaded" system.

Note that the analogy with optoelectronics and wavegrating interaction needs some care: a reflection event in 
Table 1. Uses of the Dark State Terminology: Domain, Relevant References, Comment

\begin{tabular}{|c|c|c|}
\hline Domain $^{a}$ & References & Comments $^{b}$ \\
\hline Coherent population trapping & {$[1-10]$} & $\begin{array}{l}\text { Initially for archetypal systems such as } \Lambda \text { and } V \\
\text { three-levels configurations, with two strong laser } \\
\text { beams. } \\
\text { Dark states are those dressed states formed } \\
\text { through transitions that, in the presence of the } \\
\text { second beam, do not absorb the first beam; bright } \\
\text { states are the remaining radiating transitions. }\end{array}$ \\
\hline Electromagnetically induced transparency & {$[14-18]$} & $\begin{array}{l}\text { Derived from CPT but one beam has low power } \\
\text { and behaves as a linear probe; can be seen as } \\
\text { nonlinear resonant index changes. Here, dark } \\
\text { states are also dressed states that do not absorb } \\
\text { the incoming energy whereas their constituent } \\
\text { states would, in the low power linear regime. } \\
\text { Bright states are the other dressed states. }\end{array}$ \\
\hline Superradiance, Dicke states & {$[21,22]$} & $\begin{array}{l}\text { Coherent interaction of, e.g., emitting two-level } \\
\text { atoms. Bright states are the fully symmetrical } \\
\text { states of an ensemble of identical atoms (e.g., } \\
\text { two-level atoms, not all in the same state). Other } \\
\text { linear combinations give rise to various degrees of } \\
\text { radiant states. There are notably subradiant } \\
\text { states. The less radiant are termed dark states, } \\
\text { notably in quantum optics and quest for best } \\
\text { possible qubits. }\end{array}$ \\
\hline
\end{tabular}

Excitons

$[19,20,23]$

Transition of complex excited state

Plasmonics

$[33,38-40]$

Waveguide dark mode

Domains not relevant

Dark Hamiltonian

Degraded molecules (no reference given)
In [23] a microcavity mode couples to nearly degenerate exciton subsets, only two bright modes arise, the rest are dark.

Between molecular energy surfaces in the FranckCondon picture, or in highly excited molecules, [28] is specific of Rydberg atom.

Plasmonic modes poorly coupled to the outside are called dark.

Combination of waveguide modes does not interact with cavity and can be termed dark, for example.

Dark states refer to Hamiltonian of particles without interaction with light, hence a kinetic term $p^{2}$ and not $(p-q A)^{2}$.

Generally chemically reacted, not directly relevant here. Some cases may, however, correspond to the transition of complex excited states, but the material aspect dominates over its internal level couplings

\footnotetext{
${ }^{a}$ The last two entries are considered as not relevant in our discussion.

${ }^{b} \mathrm{~A}$ short definition of dark and bright states is given in most entries.
}

a standard distributed periodic dielectric structure [such as distributed Bragg reflector (DBR) mirrors and distributed feedback (DFB) diodes] is actually a coupling. And now, a paradox creeps in if we consider that the absence of such a coupling - the situation in the transmission window of a DBR-represents a dark case (all reflective interfaces do not give a coherent response); conversely a bright case arises in the reflective stop band, when transmission vanishes and interaction is effective. We hope that on further reading the significance of these remarks will be clear and helpful.

An extra difficulty in the dark-bright distinction is to appreciate which are the "canonical" ways to excite the system, and which are not canonical. When a grating written along a guiding structure couples an entrance mode to an internal mode not detected at a standard exit [42], it is not clear, depending on the configuration, whether reflection is bright and transmission dark or the 
contrary. Some internal modes in coupled waveguide and cavities, or in photonic bandgap materials, are notoriously difficult to linearly excite, often for symmetry reasons (spontaneous emission of embedded species can help, then, see [43]). Similarly, in atom physics, the coherent excitation of several atoms with nontrivial phases is considered as delicate in most experiments, as is discussed at length in the huge body of ongoing quantum information studies. The issue that we first want to deepen is therefore "why does it happen that some eigenmodes do not see the strong interaction of their isolated constituents (uncoupled modes) when two families of modes interact?"

\section{MATRIX FORM AND TRENDS FROM THE SECULAR EQUATION}

The generic matrix describing the interaction has the form

$$
\begin{gathered}
\mathbf{M}=\left(\begin{array}{cc}
\mathbf{P} & \mathbf{G}_{p q} \\
\mathbf{G}_{q p} & \mathbf{Q}
\end{array}\right), \quad \mathbf{P}=\left(\begin{array}{lll}
E_{1} & & 0 \\
& \ddots & \\
0 & & E_{n}
\end{array}\right), \\
\mathbf{Q}=\left(\begin{array}{ccc}
E_{p+1} & & 0 \\
& \ddots & \\
0 & & E_{p+q}
\end{array}\right) .
\end{gathered}
$$

The coupling terms are ad hoc in the "full" G matrices. It is fully analogous to a Hamiltonian, but in the case of optical modes, complex diagonal terms may be introduced to represent power losses and leakages. In coupled-mode theory (CMT) [11-13,44] the diagonal terms would be propagation constants of uncoupled modes, and $\mathbf{G}$ would correspond to the coupling constants usually denoted $\kappa$ and typically induced by a dielectric constant periodic modulation. As a reminder, the rotating-wave approximation for atoms employs similar Hamiltonians, with nonHermitian terms related to loss of population [3].

A good basis for our goal is [23], a paper on vacuum Rabi splitting in microcavities with excitons, considering the coupling of a single cavity mode ( $p=1$ for us) at $E_{o}$ with a set of inhomogeneous exciton-atom modes $\left\{E_{1}, \ldots, E_{q}\right\}$, hence $N=1+N_{q}$ (=1+n in their notations), and $\mathbf{G}_{p q}=\left[g_{1}, \ldots, g_{q}\right]$ is a line vector, with all $g_{k}$ elements bounded to some value $g$ (as mentioned, $[26,27]$ studied the same matrix). Their main result states that, for coupling strengths larger than the typical separation of the inhomogeneous set $\left\{E_{1}, \ldots, E_{q}\right\}, N_{b}=2$ bright states capitalize most of the coupling. This results in the so-called Rabi splitting being $E_{ \pm}=E_{o} \pm\left(\sum g_{k}^{2}\right)^{1 / 2}$ (becoming $g \sqrt{q}$ if for $k=1 \ldots j, g_{k}=g$ and $\left.\hbar \equiv 1\right)$. The $N_{d}=N-2=N_{q}-1$ other states are affected only to second order in $g$, depending on the exact separations among the $\left\{E_{1}, \ldots, E_{q}\right\}$ set. The authors of [23] extend their study to imaginary parts of the energies, to grasp the expected absorption mechanisms, thus confirming the "darkness" of the $N-2$ states, and, further, the robustness of the linewidth of vacuum Rabi split lines against appreciable inhomogeneity of the excitonic (electronic) part of the system under study.

Some insight may be gained from the determinant $\operatorname{Det}(\mathbf{M}-\lambda \mathbf{1})$, since it appears at the denominator of all lin-

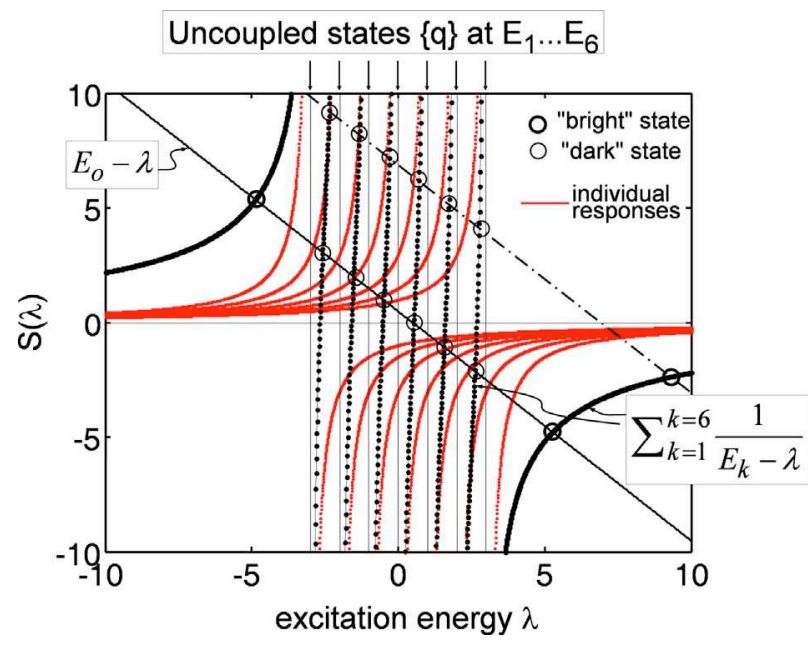

Fig. 1. (Color online) Graphical solution of Eq. (2). The contributing homographic functions are thinner continuous dark gray lines, and each represents the response of a single oscillator. Solutions associated to bright and dark states are shown for a small $E_{o}$ value (dashed line) and for a more detuned value (dasheddotted line)

ear excitation problems (and of course its zeroes are the eigenvalues). Reference [23] shows that within a nonzero factor, the secular equation $\operatorname{Det}(\mathbf{M}-\lambda \mathbf{1})=0$ simply reads

$$
S(\lambda)=\sum_{k=1}^{q} g_{k}^{2}\left(E_{k}-\lambda\right)^{-1}-\left(E_{o}-\lambda\right)=0 .
$$

The plot of Fig. 1 illustrates the graphical solution of $S(\lambda)=0$ : the $q$ homographic functions $g_{k}^{2}\left(E_{k}-\lambda\right)^{-1}$ have their singularities clustered around $\left\langle\left\{E_{q}\right\}\right\rangle$, and cross zero once between each of them (they are nondegenerate). Conversely, far from the singularities, their asymptotic branches add up constructively. The crossings with the solid line $S_{o}(\lambda)=E_{o}-\lambda$ (case $E_{o} \sim\left\langle\left\{E_{q}\right\}\right\rangle$ ) lie in majority close to the central zeroes, in which case original modes contribute to the linear response with a combination of phase retardation and advance $\left[\left(E_{k}-\lambda\right)^{-1}\right.$ have variable signs], that is, a set of dark modes form, which are intercalated between original ones [27] (and arbitrarily clustered if the initial states are sufficiently close to perfect degeneracy). Conversely, the two bright modes correspond to the two intersections on the sides. There, the situation for all $g_{k}^{2}\left(E_{k}-\lambda\right)^{-1}$ implies, for a given intersection, a single sign of all responses, hence a collective coupling. The situation for a typical detuned case (dashed-dotted line, $\left.\left|E_{o}-\left\langle\left\{E_{q}\right\}\right\rangle\right|>\sim g \sqrt{q}\right)$ shows that the coupling effects are comparatively weak, the eigenvalues are now close to the uncoupled values, with more perturbative shifts.

\section{DISPERSION FOR TWO FAMILIES $\left(N_{F}=2\right)$}

This first step in understanding the elementary diagonalization now obviously leads to the situation of Figs. 2(a) and $2(\mathrm{~b})$, if some spatial dispersion parametrizes the two families (other parameters such as static fields for atomic spectra splitting may be devised). In the case of Fig. 2(a) $E_{o}$ is nondispersive while the $\left\{E_{q}\right\}$ represent forward modes with some common group index $n_{g, k}=n_{g}$. It follows 

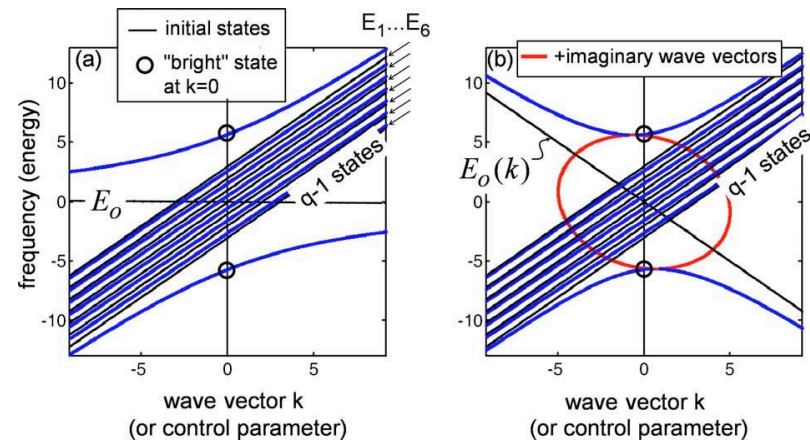

(or control parameter)

Fig. 2. (Color online) Dispersion relation for one mode coupled to a manifold of $\{q\}$ modes; (a) the single mode $\{p=1\}$ is dispersionless; (b) the single mode $\{p=1\}$ has backward dispersion; The ellipse-shaped line is the sum of real and imaginary wave vectors as is classical in the CMT approach.

from the above that two bright modes describe a large anticrossing pair of branch, while the $N_{d}=N_{q}-1=N-2$ remaining branches manage to go through the interaction region with only a faint slowdown, on a scale dictated by the interplay of the local interbranch spacing $E_{k}-E_{k \pm 1}$ and the scale of the coupling region (as can be inferred also from Fig. 1). Figure 2(b) is just the same case, but with the $E_{o}$ branch tilted (backward mode), thus resembling [35]. The single dark mode in [35] is the one not affected by the anticrossing, as before.

At this stage, a further precaution regards the analogy between CMT and Hamiltonians. The usual way to employ CMT in media without gain makes use of the frequency as the control parameter in order to find the propagation constants as eigenvalues. The matrix of interest is not Hermitian, but symmetric. There is a kind of duality, however, if the wave vector is formally considered as the control parameter, a view that circumvents the change of nature of the eigenvalues in the gaps that occurs in standard CMT.

Along another line, before proceeding, we note that the dark mode offers easy propagation, and no interaction, thanks to the "loading" of uncoupled modes with the right phase and amplitude combination. Whether such a combination is easy or difficult to reach from external excitation is a generic coupling issue, generally a demanding feat for isolated atoms, maybe a less demanding one for optical beams. We would like now to remark that in a DBR, in relation with the role of loading internal modes on the overall response, the presence of the transmission window in such a coherent treatment should be seen as the "nontrivial" feature, since in isolation, each period reflects a fair amount. It is only collectively that the internal degrees of freedom (local forward and backward waves) form a Bloch mode allowing nearly perfect transmission. With this in mind, it is the stopband that genuinely hits the bright mode of the DBR, because the interaction is perfectly in phase for all reflections.

In Fig. 3, we generalize the above results to two multiple families (i.e., $N_{p}>1$ now). In Figs. 3(a) and 3(b) we scan cases analogous to those of Figs. 2(a) and 2(b). The added complexity of the first family does not change much the picture, there are still $N_{p}=2$ bright modes in our case. In Figs. 3(c) and 3(d) we zoom on the interaction among the dark states. The intricate net of modes in the center
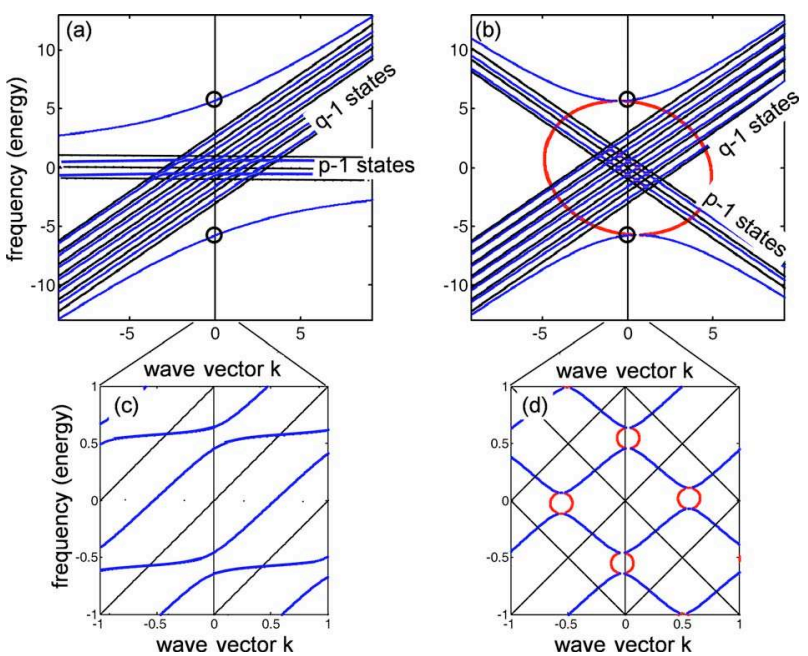

Fig. 3. (Color online) Dispersion relation for a general case between $\{p\}$ and $\{q\}$ modes; (a) $\{p\}$ modes are dispersionless; (b) $\{p\}$ modes have backward dispersion; (c) and (d) are magnifications of the indicated regions of (a) and (b) showing the local advent of slow light flatbands.

may now exhibit some degree of slowness on the whole scale covered by the uncoupled branches. This scale is much larger than the scale of a single anticrossing. Unfortunately, we are not aware of a case offering an analytical solution or a simple secular equation in such a case. However, the linear response for the excitation at a particular frequency or wave vector may now become quite complex, with as many sharp features-analogous to "induced transparency" windows-as anticrossings.

\section{GENERAL CASE, DISPERSION FOR $N_{F}>2$ FAMILIES}

Furthermore, we can still generalize (without mathematical demonstration) and reach a classification: the general case is to consider $F$ families of modes having no internal interaction, but only external ones. The $\mathbf{G}$ matrices are then to be defined among each of the $N_{F}\left(N_{F}-1\right) / 2$ family pairs. In the above, $N_{F}=2$, and only one kind of $\mathbf{G}$ matrix was to be defined. In the general case, we can infer that there are $F$ bright states and $N_{d}=N-N_{F}$ dark states. In [34], $N_{F}=3$ and $N=5$ states are present (two waveguides with degenerate forward and backward modes, plus a nondegenerate cavity or CROW mode), hence $N_{d}=5-3$ $=2$ dark states are formed. We propose to term this "an $N-N_{F}$ classification," in order to compare the numerous emerging proposals with a basic synthetic tool. $N_{F}=3$ can often be met with bidirectional waveguides and more localized modes, as discussed early enough [45], and revived in many recent studies.

It remains to be proven that imaginary parts of the eigenvalues, $\lambda^{\prime \prime}$, generally follow the same trends as in [23] (and also of the illustration in [19] for instance), but an examination of the reasons for the association of strong splitting and strong absorption ("brightest" states) appears from elementary algebra from Eq. (2): peaks in $\lambda^{\prime \prime}$ also tend to follow the algebraic sum of the strength of homographic functions. However, details of coupling to the bath allowing such a relaxation would be needed for more firm physical conclusions. 

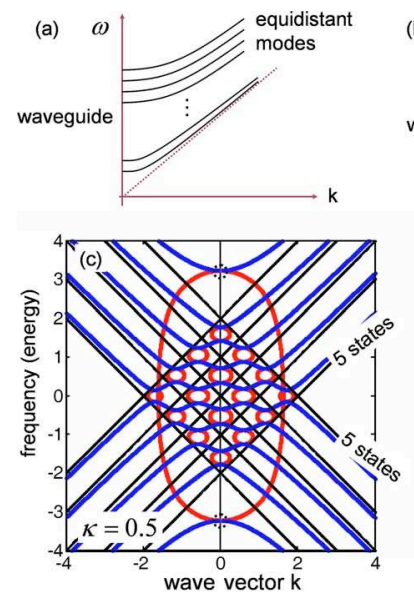
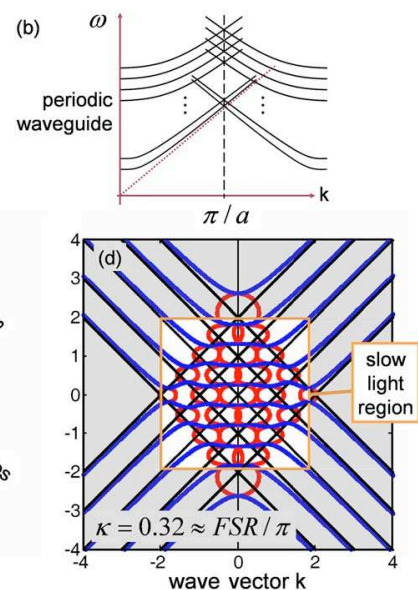

Fig. 4. (Color online) (a) Dispersion of an ideal straight broad waveguide (hyperbolic branches); (b) same with folding at Brillouin zone edge; (c), (d) dispersion for $5+5$ modes, with the indicated coupling. The closed curves, more intricate in (d), are again the sum of real plus imaginary parts of wave vectors in the coupled mode (CMT) approach. Case (d) shows maximal light slowdown in the indicated region.

\section{RELATION WITH SLOW MODES IN A MULTIMODE CASE}

As a last point, the case of Figs. 3(b) and 3(d) is nothing but contradirectional coupling by a grating of period $a$ in a multimode waveguide at the Brillouin zone boundary $[41,46]$. In this precise case of a broad waveguide, the branches are hyperbolic and at the Brillouin zone edge $(k=\pi / a)$, they are locally linear and equidistant with some local free spectral range (FSR) [Figs. 4(a) and 4(b)], leaving the number of useful parameters to two (FSR and $g$ insofar as $N$-independent physics is sought).

To link this point to our previous work, we may now consider what happens for decreasing interactions. Figures 4(c) and 4(d) show that within the lozenge-shaped region of darkness, the advent of slow light is maximal for a critical coupling condition $\kappa \equiv g=0.32$, just around the point where two split bright states start to emerge separately from the dark ones. We have discussed in [41] the occurrence of maximally slowed down light for a coupling constant $g \equiv \mathrm{FSR} / \pi=0.318$ here, and the advent of bright modes that take most of the coupling strength beyond this value. Performing novel optical functions with dark modes is thus promising in several respects, again with sufficient care for coupling, boundary conditions, and of course losses.

\section{CONCLUSION}

In summary, we have discussed that the interaction of $N$ modes grouped in $N_{F}$ families gives $N_{d}=N-N_{F}$ dark modes and $N_{b}=N_{F}$ bright modes, whose interaction occurs more collectively than the others. The location of dark modes in a generic band diagram has been discussed for $N_{F}=2$, when two families interact and form two bright states and $N-2$ dark states. The significance to related topics (light-matter interaction, imaginary parts, physics of eigenmodes in DBR) has also been given and possible paradoxes or semantic caveats addressed. We believe that this discussion helps in distinguishing and classifying among emerging proposals, be it simply by terming the interaction scheme an " $N-N_{F}$ " scheme; e.g., $N-N_{F} \equiv 5$ -3 in [34]; $N-N_{F} \equiv n-1$ in [23], etc. Grating-induced back reflection in a broad multimode waveguide also falls within the scope of the present description on the optical side. But because our discussion is generic, we note that it could also apply to electronic and acoustic dispersion effects. The analogy of CPT and the coupled-mode theory of coupled rings is rendered quite visually on the cover of the IEEE-LEOS December 2008 newsletter and in Fig. 3 of [47].

\section{ACKNOWLEDGMENTS}

The author acknowledges the highly valuable help of $R$. Houdré in setting up the general discussion, as well as useful hints from A. Badolato and A. Imamoglu.

\section{REFERENCES}

1. F. T. Hioe and C. E. Carroll, "Coherent population trapping in $N$-level quantum systems," Phys. Rev. A 37, 3000-3005 (1988).

2. F. T. Hioe and J. H. Eberly, " $N$-Level coherence vector and higher conservation laws in quantum optics and quantum mechanics," Phys. Rev. Lett. 47, 838-841 (1981).

3. J. R. Ackerhalt, J. H. Eberly, and B. W. Shore, "Statistical broadening and population loss in strongly excited threelevel systems," Phys. Rev. A 19, 248-263 (1979).

4. K. A. Shore, "Control of spontaneous emission in microcavity laser diodes," Physica B 175, 123-126 (1991).

5. G. Alzetta, A. Gozzini, L. Moi, and G. Orriols, "An experimental method for the observation of $\mathrm{rf}$ transitions and laser beat resonances in oriented $\mathrm{Na}$ vapour," Nuovo Cimento Soc. Ital. Fis., B 36, 5-20 (1976).

6. G. Alzetta, L. Moi, and G. Orriols, "Nonabsorption hyperfine resonances in a sodium vapour irradiated by a multimode dye-laser," Nuovo Cimento Soc. Ital. Fis., B 52 , 209-218 (1979).

7. E. Arimondo and G. Orriols, "Nonabsorbing atomic coherences by coherent two-photon transitions in a threelevel optical pumping," Lett. Nuovo Cimento Soc. Ital. Fis. 17, 333-338 (1976).

8. H. R. Gray, R. M. Whitley, and C. R. Stroud, Jr., "Coherent trapping of atomic populations," Opt. Lett. 3, 218-220 (1978).

9. E. Arimondo, "Coherent population trapping in laser spectroscopy," in Progress in Optics, E. Wolf, ed. (Elsevier, 1996), Vol. XXXV, pp. 257-354.

10. B. Shore, M. Johnson, K. C. Kulander, and J. Davis, "The Livermore experience: contributions of J. H. Eberly to laser excitation theory," Opt. Express 8, 28-43 (2001).

11. H. Kogelnik and C. V. Shank, "Coupled wave theory of distributed feedback lasers," J. Appl. Phys. 43, 2327-2335 (1972).

12. T. Tamir, "Guided wave optoelectronics," in Springer Series in Electronics and Photonics (Springer, 1990).

13. A. Yariv, "Coupled mode theory for guided wave optics," IEEE J. Quantum Electron. 9, 919-933 (1973).

14. A. Imamoglu and S. E. Harris, "Lasers without inversion: interference of dressed lifetime-broadened states," Opt. Lett. 14, 1344-1346 (1989).

15. S. E. Harris, "Lasers without inversion: interference of lifetime broadened resonances," Phys. Rev. Lett. 62, 1033-1036 (1989).

16. H. Schmidt and A. Imamoglu, "Giant Kerr nonlinearities obtained by electromagnetically induced transparency," Opt. Lett. 21, 1936-1938 (1996).

17. D. Budker, D. F. Kimball, S. M. Rochester, and V. V. Yaschuk, "Nonlinear magneto-optics and reduced group 
velocity of light in atomic vapor with slow ground state relaxation," Phys. Rev. Lett. 83, 1767-1770 (1999).

18. K. J. Boller, A. Imamoglu, and S. E. Harris, "Observation of electromagnetically induced transparency," Phys. Rev. Lett. 66, 2593-2596 (1991).

19. G. C. La Rocca, F. Bassani, and V. M. Agranovich, "Biexcitons and dark states in semiconductor microcavities," J. Opt. Soc. Am. B 15, 652-660 (1998).

20. M. Nirmal, D. J. Norris, M. Kuno, M. G. Bawendi, A. L. Efros, and M. Rosen, "Observation of the 'dark exciton' in CdSe quantum dots," Phys. Rev. Lett. 75, 3728-3731 (1995).

21. R. H. Dicke, "Coherence in spontaneous radiation processes," Phys. Rev. 93, 99-110 (1954).

22. S. Haroche, "Superradiance: an essay on the theory of collective spontaneous emission,” Phys. Rep. 93, 301-396 (1982).

23. R. Houdré, R. P. Stanley, and M. Ilegems, "Strong coupling régime in the presence of inhomogeneous broadening: resolution of an homogeneous linewidth in an inhomogeneously broadened system," Phys. Rev. A $\mathbf{5 3}$ 2711-2715 (1996).

24. R. Houdré, C. Weisbuch, R. P. Stanley, U. Oesterle, P. Pellandini, and M. Ilegems, "Measurement of cavitypolaritons dispersion curve from angle resolved photoluminescence experiments," Phys. Rev. Lett. 73, 2043-2046 (1994).

25. C. Weisbuch, M. Nishioka, A. Ishikawa, and Y. Arakawa, "Observation of the coupled exciton-photon mode splitting in a semiconductor quantum microcavity," Phys. Rev. Lett. 69, 3314-3317 (1992).

26. S. L. Coy, R. Hernandez, and K. K. Lehmann, "Limits on transitions to Gaussian orthogonal ensemble behaviour: saturated radiationless transitions between strongly coupled potential surfaces,” Phys. Rev. A 40, 5935-5949 (1989).

27. S. L. Coy and K. K. Lehmann, "Energy-level statistics for a relaxation Hamiltonian," Phys. Rev. A 36, 404-407 (1987).

28. M. V. Federov and M. Y. Ivanov, "Coherence and interference in a Rydberg atom in a strong laser field: excitation, ionization, and emission of light," J. Opt. Soc. Am. B 7, 569-572 (1990).

29. A. I. Streltsov, N. V. Dobrodey, and L. S. Cederbaum, "Charge transfer effects in molecule-negative ion complexes induced by core ionization," J. Chem. Phys. 119, 3051-3062 (2003).

30. S. Keshavamurthya, N. R. Cerruti, and S. Tomsovic, "Analyzing intramolecular vibrational energy redistribution via the overlap intensity-level velocity correlator," J. Chem. Phys. 117, 4168-4177 (2002).

31. R. Thissen, P. Lablanquie, R. I. Hall, M. Ukai, and K. Ito, "Photoionization of argon, krypton and xenon clusters in the inner valence shell region," Eur. Phys. J. D 4, 335-342 (1998)

32. L. Duckhwan and A. C. Albrecht, "On the interaction operator in the optical spectroscopies," J. Chem. Phys. 78, 3382-3392 (1983).
33. M. Wegener, J. L. Garcia-Pomar, C. M. Soukoulis, N. Meinzer, M. Ruther, and S. Linden, "Toy model for plasmonic metamaterial resonances coupled to two-level system gain," Opt. Express 16, 19785-19798 (2008).

34. P. Chak, J. K. S. Poon, and A. Yariv, "Optical bright and dark states in side-coupled resonator structures," Opt. Lett. 32, 1785-1787 (2007)

35. H. C. Liu and A. Yariv, "Grating induced transparency (GIT) and the dark mode in optical waveguides," in Lasers and Electro-Optics, 2008 OSA Technical Digest Series (Optical Society of America, 2008), paper CMK5.

36. A. Yariv, Y. Xu, R. K. Lee, and A. Scherer, "Coupledresonator optical waveguide: a proposal and analysis," Opt. Lett. 24, 711-713 (1999).

37. E. Waks and J. Vuckovic, "Coupled mode theory for photonic crystal cavity-waveguide interaction," Opt. Express 13, 5064-5073 (2005).

38. H. Mertens, A. F. Koenderink, and A. Polman, "Plasmonenhanced luminescence near noble-metal nanospheres: comparison of exact theory and an improved Gersten and Nitzan model," Phys. Rev. B 76, 115123 (2007).

39. D. J. Bergman and M. I. Stockman, "Surface plasmon amplification by stimulated emission of radiation: quantum generation of coherent surface plasmons in nanosystems," Phys. Rev. Lett. 90, 027402 (2003).

40. J. E. Kihm, Y. C. Yoon, D. J. Park, Y. H. Ahn, C. Ropers, C. Lienau, J. Kim, Q. H. Park, and D. S. Kim, "Fabry-Perot tuning of the band-gap polarity in plasmonic crystals," Phys. Rev. B 75, 035414 (2007).

41. H. Kurt, H. Benisty, T. Melo, O. Khayam, and C. Cambournac, "Slow-light regime and critical coupling in highly multimode corrugated waveguides," J. Opt. Soc. Am. B 25, C1-C14 (2008).

42. E. Peral, A. Yariv, and L. Fellow, "Supermodes of gratingcoupled multimode waveguides and application to mode conversion between copropagating modes mediated by backward Bragg scattering," J. Lightwave Technol. 17, 942-947 (1999).

43. H. Benisty, C. Weisbuch, D. Labilloy, M. Rattier, C. J. M. Smith, T. F. Krauss, R. M. DeLaRue, R. Houdré, U. Oesterle, and D. Cassagne, "Optical and confinement properties of two-dimensional photonic crystals," J. Lightwave Technol. 17, 2063-2077 (1999).

44. S. Olivier, H. Benisty, C. Weisbuch, C. J. Smith, T. F Krauss, and R. Houdré, "Coupled-mode theory and propagation losses in photonic crystal waveguides," Opt. Express 11, 1490-1496 (2003).

45. H. A. Haus and Y. Lai, "Narrow-band distributed feedback reflector design," J. Lightwave Technol. 9, 754-760 (1991).

46. O. Khayam, C. Cambournac, H. Benisty, M. Ayre, H. Brenot, G. H. Duan, and W. Pernice, "In-plane Littrow lasing of broad photonic crystal waveguides," Appl. Phys. Lett. 91, 041111 (2007).

47. L. Zhang, M. Song, T. Wu, L. Zou, R. G. Beausoleil, and A. E. Willner, "Embedded ring resonators for microphotonics applications," Opt. Lett. 33, 1978-1980 (2008). 